\title{
REVISIÓN TEÓRICA DEL ENTRENAMIENTO CON RESTRICCIÓN DEL FLUJO SANGUÍNEO HACIA LA HIPERTROFIA Y LA FUERZA MUSCULAR.
}

\author{
THEORETICAL REVIEW OF TRAINING WITH RESTRICTION OF BLOOD FLOW \\ TOWARDS HYPERTROPHY AND MUSCLE STRENGTH.
}

Cómo citar: Fuquen, D. y Gracia, Á. (2019). Revisión teórica del entrenamiento con restricción del flujo sanguíneo hacia la hipertrofia y la fuerza
muscular. R. Actividad fis. y deporte. 5 (2): 142-170. Artículo de acceso abierto publicado por: Revista Digital: Actividad Física y Deporte, bajo una licencia Creative Commons CC BY-NC 4.0

\section{Daniel Alejandro Fuquen Rivas}

Profesional de Ciencias del deporte de la Universidad de Ciencias Aplicadas y Ambientales U.D.C.A.

E-mail: danielfu13@hotmail.com

\section{Álvaro José Gracia Díaz}

Dr. (Ph. D). En Pedagogía y Didáctica de la Educación

Física. Magister en Docencia Universitaria.

Especialista en Teoría, Métodos y Técnicas de la investigación social. Profesor de la Universidad de Ciencias Aplicadas y Ambientales (U.D.C.A.).

\section{RESUMEN}

Introducción: Esta monografía realiza una recopilación de información de las bases de datos, así como de artículos escritos sobre el entrenamiento en oclusión, con la finalidad de desarrollar la fuerza y la hipertrofia muscular. Objetivo general: Describir los procesos de hipertrofia y de fuerza mediante el uso del entrenamiento con restricción de flujo sanguíneo. Metodología: Para esta investigación prevalece el enfoque cualitativo, mientras que, el diseño es transversal, porque se hace la recopilación y análisis de la información en un sólo momento, en tanto que el alcance de la investigación es exploratorio, debido a que, se encontraron 60 artículos todos de origen internacional (Hernández, R. y Mendoza, C., 2008). Las bases de datos en que se encontraron los artículos funcionales fueron: ProQuest (12), ScienceDirect (8), SportDiscus (15), Scopus (12) y PubMed (13). Resultados: Los artículos que se exponen en la tabla los principales autores del tema, donde se indica el objetivo y el aporte. Los demás artículos se traduce el título al español y se da una categoría según el tema central, los cuales son: Adaptaciones, Efectos, Hipertrofia, Seguridad y daños, Salud, Hormonal, Neuronal, y Deportes. Conclusión: el entrenamiento de restricción de flujo sanguíneo tiene como principal efecto la hipertrofia muscular, $y$, debido al agrandamiento de las fibras musculares, conlleva a la ganancia de fuerza. Sin embargo, aún faltan más estudios que complementen con un respaldo científico a nivel morfológico y fisiológico el proceso de hipertrofia con este método.

Palabras clave: Hipertrofia, Fuerza, Entrenamiento, Oclusión Vascular. 


\section{ABSTRACT}

Introduction: This monograph compiles information from databases, as well as written articles about occlusion training, in order to develop strength and muscle hypertrophy. Course objective: To describe the processes of hypertrophy and strength through the use of training with restriction of blood flow. Methodology: For this research the qualitative approach prevails, while the design is transversal, because the collection and analysis of the information is done in a single moment, while the scope of the research is exploratory, because, they were found 60 articles all of international origin (Hernández, R. and Mendoza, C., 2008). The databases in which the functional articles were found were: ProQuest (12), ScienceDirect (8), SportDiscus (15), Scopus (12) and PubMed (13). Results: The articles that are exposed in the table the main authors of the topic, where the objective and the contribution are indicated. The other articles are translated into Spanish and a category is given according to the central theme, which are: Adaptations, Effects, Hypertrophy, Safety and Damage, Health, Hormonal, Neuronal, and Sports. Conclusion: the training of restriction of blood flow has as main effect the muscular hypertrophy, and, due to the enlargement of the muscular fibers, it leads to the gain of strength. However, there are still more studies that complement the process of hypertrophy with this method with a scientific support at a morphological and physiological level.

Key words: Hypertrophy, Strength, Training, Vascular Occlusion.

\section{INTRODUCCIÓN}

El entrenamiento de fuerza ha demostrado ser efectivo con cargas que superen el 70\% del 1 RM (Ratamess, Albar, Evetoch, Housh, Kibler, Kraemer, y Triplett, 2009), pudiendo producir a su vez hipertrofia muscular.

La hipertrofia muscular no solo es usada para fines estéticos como en competiciones de fisicoculturistas y fitness, también se ha demostrado que la mayoría de lesiones van acompañadas por atrofia, por tanto, el entrenamiento de fuerza enfocado en aumentar la masa muscular, busca evitar lesiones y ser de ayuda en rehabilitación para aquellos que ya sufrieron lesiones (Manimmanakorn, Hamlin, Ross, Taylor, y Manimmanakorn, 2013).

En los últimos años hay un entrenamiento que goza de creciente popularidad, debido a la hipertrofia como resultado principal que induce ejercitándose bajo presión de una extremidad, alcanzando o superando la tensión arterial sistólica (Warnock y Bachman, 2015). Este tipo de entrenamiento fue creado en Japón por Yoshiaki Sato denominándolo como KAATSU y se dio a conocer en 1985. Sin embargo, en el Occidente es a menudo denominado "entrenamiento oclusivo" o "entrenamiento de restricción de flujo sanguíneo" (Mohamed, Basant, y Larion, 2017).

Se ha demostrado que el entrenamiento de resistencia de baja carga (20-50\% 1 RM) con restricción del flujo sanguíneo mejora el tamaño y la función muscular en una variedad de poblaciones, por ejemplo, Takarada et al. (2002) demostraron un aumento del $14 \%$ en la fuerza del extensor de rodilla de los sujetos jóvenes cuando se combinó el entrenamiento de resistencia con poca carga a una intensidad del 50\% $1 \mathrm{RM}$ con 
la restricción del flujo sanguíneo en comparación con ningún cambio en la fuerza con el entrenamiento de resistencia tradicional. De hecho, se han realizado algunas observaciones de aumentos en la fuerza como resultado de la hipertrofia muscular, después de la restricción del flujo sanguíneo en escalas de tiempo cortas, como 2 semanas (Abe et al. 2005).

Este método se fundamenta en las respuestas hormonales y anabólicas que resultan al entrenar un músculo bajo condiciones de isquemia (falta de riego sanguíneo e hipoxia). Para restringir temporalmente el riego sanguíneo hacia el músculo se utiliza un manguito de oclusión que funciona como torniquete en la extremidad a trabajar. Es importante que la presión sea regulada mediante estos dispositivos para poder controlar la presión, valores que oscilan de 100 a $200 \mathrm{mmHg}$ (Barnett, Dankel, Counts, Nooe, Abe, Loenneke 2016).

La oclusión vascular permite obtener un gran estímulo anabólico en los músculos constreñidos sin necesidad de emplear grandes pesos, alrededor del 20\% del $1 \mathrm{RM}$. (Yasuda, T., Loenneke, P., Thiebaud, S., \& Abe, T., 2012).

Los autores Mohamed, Basant, y Larion, determinan que el entrenamiento de restricción de flujo sanguíneo tiene una capacidad de aumentar la fuerza y la masa muscular en sujetos de edades y de condiciones físicas dispares, (Mohamed, Basant, y Larion, 2017), por lo que no solo ha sido usado en pacientes de la tercera edad, sino también con el objetivo de prevenir la atrofia muscular en pacientes renales 0 pacientes que se encuentran en rehabilitación luego de una intervención quirúrgica.
Aunque la investigación en el área del entrenamiento con restricción del flujo sanguíneo ha aumentado en interés, los estudios hasta el momento han utilizado diferentes protocolos que dificultan el establecimiento de un régimen de entrenamiento óptimo. Esto es especialmente cierto para las cargas de trabajo utilizadas durante el ejercicio, ya que los investigadores utilizan cargas que oscilan entre 20 y $80 \% 1$ RM (Laurentino et al. 2008, Reeves et al. 2006).

Abe et al. (2005) investigaron los efectos del entrenamiento de resistencia de baja intensidad dos veces al día (20\% de 1-RM) con restricción del flujo sanguíneo venoso en IGF1 (factor de crecimiento insulínico) y tamaño del músculo esquelético. El grupo de entrenamiento de restricción de flujo sanguíneo vascular de baja intensidad se comparó con un grupo de entrenamiento de baja intensidad (20\% de $1-\mathrm{RM})$ sin alguna restricción del flujo de sangre. Hubo un aumento significativo en el IGF-1 circulante y el área de sección transversal muscular en el grupo de restricción del flujo sanguíneo vascular de baja intensidad, pero no en el grupo de baja intensidad. Otros dos aspectos importantes de este estudio son que las ganancias de hipertrofia se observaron en dos semanas y los marcadores de daño muscular (mioglobina, CPK y peróxido de lípidos) no fueron elevados. Esto indica que el entrenamiento de restricción del flujo sanguíneo vascular puede producir hipertrofia muscular en un corto período de tiempo, produce hipertrofia similar al entrenamiento de alta intensidad y no eleva los marcadores de daño muscular. 


\section{DESCRIPCIÓN GENERAL DEL} PROYECTO

De esta manera, el presente trabajo se denomina revisión bibliográfica, debido a que se realiza una búsqueda de información proveniente de las bases de datos ProQuest (12), ScienceDirect (8), SportDiscus (15), Scopus (12) y PubMed (13), acerca del entrenamiento de restricción de flujo sanguíneo con la finalidad de determinar los mecanismos que inciden en el incremento de la masa muscular y la fuerza usando el entrenamiento oclusivo como principal medio. Luego de encontrar y determinar 60 artículos funcionales internacionales, se describe a nivel morfológico, fisiológico y físico, el proceso que se lleva acabo de manera interna durante y después del entrenamiento de restricción de flujo sanguíneo; también se muestra aquellas investigaciones en donde se expone cuáles fueron las repeticiones, la carga, las series y el descanso que fueron usadas con el método y que dieron resultados positivos, aumentando la masa muscular (proceso que se denomina hipertrofia) y la fuerza, mediante el uso de un manguito de presión con el cual ayuda a haber una restricción parcial del flujo sanguíneo (Manini, \& Clark, 2009).

\section{ALCANCE DE LA INVESTIGACIÓN.}

El presente trabajo se determina que es bibliográfico, descriptivo y de corte mixto. Esto es, debido a que los trabajos descriptivos son observacionales en los cuales no se interviene o manipula el factor de estudio, es decir se describe fenómenos, situaciones, contextos y eventos (García, 2003). Por tanto, según Hernandez, Fernández y Batista (2010), únicamente se pretende recoger información de manera independiente sobre conceptos o variables sin el objetivo de indicar el cómo se relacionan éstas.

Los trabajos bibliográficos parten del análisis de documentos, este trabajo corresponde a un estudio bibliográfico, ya que se analizan 60 artículos. Por último, el enfoque mixto aplica para este trabajo dado que la información se analiza de manera cualitativa y de manera cuantitativa se expone tanto el volumen de trabajos, como el volumen de trabajo, los porcentajes de carga, tiempos de descanso y demás. (Campo, Granados, Muñoz, Sergio, \& Rodríguez, 2012).

\section{¿Qué va a beneficiar o cambiar?}

Proponer un modelo de entrenamiento con el método de restricción de flujo sanguíneo, con el objeto de obtener hipertrofia muscular y aumento de la fuerza, teniendo como respaldo las investigaciones en las cuales imparten diversos protocolos de entrenamiento y que se obtuvieron resultados favorables.

\section{Resultados esperados}

Por lo tanto, se espera que se pueda realizar las respectivas observaciones de los diferentes artículos, que se interpreten de manera idónea, sin desviarse del objetivo y que se pueda determinar de manera clara la forma más adecuada de entrenar con este método; además, apuntar qué aspectos faltaría por investigar. 


\section{Pregunta investigativa}

¿Qué describen las diferentes investigaciones provenientes de artículos funcionales acerca del entrenamiento con restricción de flujo sanguíneo para la fuerza e hipertrofia muscular?

\section{OBJETIVOS}

\section{Objetivo general}

Describir los procesos de hipertrofia y de fuerza mediante el uso del entrenamiento con restricción de flujo sanguíneo.

\section{Objetivos específicos}

- Recolectar escritos existentes que posean contenido acerca del entrenamiento con restricción de flujo sanguíneo enfocados a la hipertrofia y la fuerza

- Determinar los beneficios del entrenamiento con restricción de flujo sanguíneo.

- Proponer una estructura metodológica de la oclusión del flujo sanguíneo para la hipertrofia muscular.

\section{Hipótesis}

El grado de efectividad del entrenamiento con restricción del flujo sanguíneo, afecta directamente el desarrollo de la hipertrofia muscular.

\begin{tabular}{|l|l|}
\hline palabras claves: & Ecuación de búsqueda: \\
\hline Blood flow restriction: & blood flow restriction + hypertrophy \\
\hline Hypertrophy: & Hypertrophy + kaatsu \\
\hline Strength: & entrenamiento en oclusión + fuerza \\
\hline Kaatsu: & Kaatsu + strength \\
\hline Entrenamiento en oclusión & \\
\hline
\end{tabular}

Tabla 1 Palabras clave y ecuaciones de búsqueda.

\begin{tabular}{|c|c|c|c|c|c|c|c|c|}
\hline $\begin{array}{c}\text { Base de } \\
\text { datos }\end{array}$ & Ecuaciones de búsqueda & Año & Artículos & Funcionales & Loc. & Reg. & Nac. & Inte \\
\hline \multirow[t]{3}{*}{ Scopus: } & $\begin{array}{c}\text { blood flow restriction + } \\
\text { hypertrophy }\end{array}$ & \multirow{3}{*}{$\begin{array}{c}2018 \\
- \\
2008\end{array}$} & 197 & 3 & 0 & 0 & 0 & 12 \\
\hline & Hypertrophy + kaatsu & & 106 & 4 & & & & \\
\hline & Kaatsu + strength & & 86 & 6 & & & & \\
\hline \multirow[t]{3}{*}{ Sport Discus: } & $\begin{array}{c}\text { blood flow restriction + } \\
\text { hypertrophy }\end{array}$ & \multirow{3}{*}{$\begin{array}{c}2018 \\
- \\
2008\end{array}$} & 7 & 5 & 0 & 0 & 0 & 15 \\
\hline & Hypertrophy + kaatsu & & 2 & 7 & & & & \\
\hline & Kaatsu + strength & & 194 & 3 & & & & \\
\hline
\end{tabular}




\begin{tabular}{|c|c|c|c|c|c|c|c|c|}
\hline \multirow[t]{3}{*}{ ProQuest: } & $\begin{array}{c}\text { blood flow restriction + } \\
\text { hypertrophy }\end{array}$ & \multirow{3}{*}{$\begin{array}{c}2018 \\
- \\
2008\end{array}$} & 2887 & 4 & 0 & 0 & 0 & 12 \\
\hline & Hypertrophy + kaatsu & & 72 & 3 & & & & \\
\hline & Kaatsu + strength & & 108 & 5 & & & & \\
\hline \multirow[t]{3}{*}{$\begin{array}{l}\text { Science } \\
\text { Direct: }\end{array}$} & $\begin{array}{c}\text { blood flow restriction + } \\
\text { hypertrophy }\end{array}$ & \multirow{3}{*}{$\begin{array}{c}2018 \\
- \\
2008\end{array}$} & 2164 & 5 & 0 & 0 & 0 & 8 \\
\hline & Hypertrophy + kaatsu & & 18 & 1 & & & & \\
\hline & Kaatsu + strength & & 22 & 2 & & & & \\
\hline \multirow[t]{3}{*}{ Pudmed: } & $\begin{array}{c}\text { blood flow restriction + } \\
\text { hypertrophy }\end{array}$ & \multirow{3}{*}{$\begin{array}{c}2018 \\
- \\
2008\end{array}$} & 138 & 7 & 0 & 0 & 0 & 13 \\
\hline & Hypertrophy + kaatsu & & 32 & 3 & & & & \\
\hline & Kaatsu + strength & & 50 & 3 & & & & \\
\hline \multicolumn{3}{|c|}{ Total } & $\begin{array}{c}6083 \\
\text { Internacion } \\
\text { al. }\end{array}$ & 60 & 0 & 0 & 0 & 60 \\
\hline
\end{tabular}

Tabla 2 Bases de datos.

\section{MARCO CONCEPTUAL}

La hipertrofia, que significa aumento del tamaño de las fibras musculares individuales, es el modo principal de crecimiento muscular. Para potenciar el crecimiento muscular se debe realizar un programa de entrenamiento de resistencia, lo que a su vez mejora la fuerza del músculo y la salud del hueso, por lo que puede mejorar el rendimiento deportivo. (Baechele, Earle, 2007) Dada la fuerte correlación entre el área de sección transversal del músculo y la fuerza muscular, el incremento de la masa muscular es un objetivo principal para aquellos deportistas que participan en disciplinas de potencia y fuerza, como el rugby, fútbol o powerlifting; o para fines estéticos como lo es el deporte del físico culturismo, donde los competidores son juzgados por la cantidad y la simetría de la masa muscular. Por lo tanto, el aumento de masa muscular tiene profundas implicaciones en una variedad de población asociadas al deporte y la salud (Schoenfeld, B, 2010).

Las recomendaciones del American College of Sports Medicine, establece que la intensidad adecuada para generar un incremento del tamaño muscular en un entrenamiento de resistencia debe superar el $70 \%$ de la repetición máxima (1 RM), generando un estímulo que desencadene procesos de activación, coordinación muscular y un ambiente hormonal completamente anabólico para posteriormente mejorar la síntesis proteica y como consecuencia, el incremento de la fuerza y la hipertrofia muscular (Martín-Hernández, Marín, y Herrero, 2011).

No obstante, hay un tipo de entrenamiento que combina el ejercicio de baja intensidad con la hipoxia para el incremento de la fuerza y el tamaño muscular. Este tipo de entrenamiento se le conoce en Japón como Kaatsu, aunque en el occidente se le conoce como entrenamiento oclusivo (occlusive training) o entrenamiento con restricción del flujo sanguíneo (blood flow 
restricted training). (Martín-Hernández, Marín, y Herrero, 2011).

El entrenamiento con oclusión vascular o restricción del flujo sanguíneo tiene su origen hace 30 años en Japón, donde Yoshiaki Sato lo popularizó sobre 1985 comercializándolo como entrenamiento KAATSU (Warnock y Bachman, 2015), por lo tanto, su mayor influencia y en donde mayor estudios se han realizado es en dicho país. "ka" significa "suma" y "atsu" significa presión, puede consistir en ejercicios normales, como sentadillas, flexiones o levantamiento de pesos ligeros, y puede durar de cinco a 30 minutos.

El entrenamiento de restricción de flujo sanguíneo provoca beneficios visibles para cualquiera, y es la hipertrofia muscular adquirida con una carga alrededor del 20\% del 1RM, muy por debajo según varios estudios para conseguir fuerza e hipertrofia (de 70\% - 90\% del 1 RM) (Manimmanakorn y col., 2013).

Una característica que destaca en este método es la utilización de un instrumento que funcione como torniquete, generando así presión para restringir de manera controlada el flujo sanguíneo de las extremidades. Esta presión, debe ser de igual o superior a la tensión arterial sistólica (Manini y Clark, 2009). Para ello, se ha recomendado el uso un monitor de presión en milímetros de mercurio en los que se regularía la presión, soliendo variar ésta entre valores de 100 a 200 mmHG (Loenneke, Fahs, Rossow, Sherk, Thiebaud, y Abe, 2012).

Lo ideal es que con estos torniquetes se pueda medir la presión, con la que se está realizando el trabajo y que se pueda controlar en todo momento para no exceder los valores de presión mencionados anteriormente, porque podría ser peligroso si la presión es alta, o ser nulo sí la presión es muy baja.
Aunque se pueda realizar con torniquetes de médico, cuerdas, cauchos, bandas elásticas como manguitos sustitutivos (Hernández, Blasco, y Herrero, 2011), se aconseja que por lo menos se realice con bandas que tengan medidor de presión por milímetros de mercurio, para que, como se dijo anteriormente tener un correcto trabajo.

También, este método puede realizarse por contracción isométrica (mantener el músculo en tensión sin movimiento alguno), este es usado por aquellos pacientes que han salido de una cirugía, debido a que, se necesita bastante tiempo de reposo de alguna articulación y, por ende, es importante las extremidades no sufran de atrofia muscular, siendo este el primer efecto cuando hay ausencia de estímulo con cargas a los músculos. (Takarada y col., 2000), o aquellas personas que por el momento tienen alguna lesión o molestia muscular, el cual requieren seguir entrenando para que su rendimiento no decrezca.

El entrenamiento de restricción de flujo sanguíneo produce el crecimiento muscular a través de dos mecanismos, El primero mediante el aumento del estrés metabólico y el segundo por la alteración en el orden de reclutamiento de las fibras musculares" (Mohamed, Basant, y Larion, 2017).

En los músculos hay dos tipos principales de fibras musculares, las de contracción rápida y las de contracción lenta (puede encontrar una gran cantidad de fibras musculares). Las fibras de contracción rápida son las fibras musculares más grandes y tienen el mayor potencial de crecimiento. Estas fibras se reclutan más recientemente durante las contracciones musculares y en su mayoría son anaeróbicas (no usan oxígeno), mientras que las fibras de contracción más lentas se reclutan primero durante las 
contracciones y son aeróbicas (usan oxígeno). (Subiela \& Torres, 2010).

El entrenamiento al restringir el flujo de sangre a los músculos, la fatiga a las fibras de contracción lenta y obliga a las fibras de contracción rápida a activarse incluso a bajas intensidades para que metabólicamente el músculo funcione de una manera similar a la que lo haría si se usara cargas pesadas, pero con el uso de cargas mucho más ligeras. (Madarame, Neya, Ochi, Nakazato Sato, y Ishii, 2008).

\section{MARCO REFERENCIAL}

\section{Antecedentes}

Actualmente, se encuentra un número de 6083 artículos en la base de datos de; ProQuest (3067), ScienceDirect (2204), SportDiscus (203), Scopus (389) y PubMed (220).

Sin embargo, artículos funcionales para este trabajo son 60, procedente de las bases de datos de la siguiente manera ProQuest (12), ScienceDirect (8), SportDiscus (15), Scopus (12) y PubMed (13), la gran mayoría con idioma principal en inglés y con autores de procedencia japonesa como: Yasuda, Yamanaka, Madarame, Neya, Nakazato, Sato. Estos artículos funcionales son para analizarlos y sistematizarlos.

- El propósito de este estudio fue investigar la efectividad de 4 semanas de entrenamiento de resistencia de baja intensidad con oclusión del flujo sanguíneo en la hipertrofia muscular de la parte superior e inferior y la fuerza muscular en jugadores de fútbol de la División IA de la Asociación Atlética Colegial Nacional. Hubo 32 sujetos de edad promedio de 19 años, que fueron asignados al azar a un grupo de oclusión o grupo de control. Los atletas realizaron 4 series de press de banca y sentadilla de la siguiente manera con o sin oclusión: 30 repeticiones de $20 \%$ de 1 RM, seguidas de 3 series de 20 repeticiones a $20 \%$ de 1RM. Entre serie y serie hubo 45 segundos de descanso; yla duración del entrenamiento fue 3 veces por semana. Los datos recogidos incluyen historia de salud, presión arterial en reposo, preentrenamiento y post-entrenamiento press de banca y sentadilla 1RM, circunferencia superior e inferior del pecho, circunferencia del brazo superior e inferior, circunferencia del muslo, altura y masa corporal. Los resultados demostraron aumentos del $1 \mathrm{RM}$ en press de banca y en sentadillas $(7,0$ y $8,0 \%$, respectivamente), y aumentos en las circunferencias del tórax superior e inferior (3 y 3\%, respectivamente).

Los autores determinan luego del estudio que el entrenamiento de oclusión podría proporcionar beneficios adicionales al entrenamiento de fuerza tradicional para mejorar la hipertrofia muscular y la fuerza muscular en atletas universitarios. (Yamanaka, Farley y Caputo, 2013).

- Yasuda, Loenneke, Thiebaud y Abe (2012) investigaron los efectos agudos y crónicos de manera concéntrica y excéntrica usando el entrenamiento de restricción de flujo sanguíneo con baja intensidad, sobre el tamaño y la fuerza muscular. Diez hombres jóvenes realizaron flexión de codo con mancuernas (curl de bíceps) con una carga del $30 \%$ del 1 RM, con cuatro series de un total de 75 repeticiones, 3 días por semana durante 6 semanas. El brazo se eligió al azar para el ejercicio de manera concéntrica y el otro para el ejercicio de 
manera excéntrica con la misma carga de ejercicio para ambos. Inmediatamente después del ejercicio, el grosor muscular de los flexores del codo aumentó de forma aguda tanto de manera concéntrica (11.7\%) como con excéntrica (3.9\%) restringiendo el flujo sanguíneo, siendo aun así mayor de manera concéntrica. Después de las 6 semanas se observaron mejoras significativamente superiores, en los valores de fuerza máxima isométrica $(8,6 \%)$ medido por electromiografía, y el área de sección transversal del brazo $(12 \%)$ y del volumen muscular $(11,7 \%)$ en el brazo que se ejercitó concéntricamente con respecto al que se ejercitó excéntricamente. Para medir el volumen muscular se realizó imágenes de resonancia magnética.

- Weatherholt, Beekley, Greer, Urtel y Mikesky investigaron las adaptaciones musculares a 40 sujetos jóvenes después de realizar un entrenamiento oclusivo durante 8 semanas, 3 veces por semana. Los 40 sujetos fueron divididos al azar en dos grupos, en donde un grupo realiza 3 series de 15 repeticiones de ejercicios de bíceps y tríceps al $20 \%$ el 1 RM mientras llevaba puesto un manguito neumático para restringir el flujo sanguíneo en un brazo, por otra parte, el grupo control realizó la misma actividad, pero sin el manguito.

Se comprobó que los brazos que se ejercitaron con restricción del flujo sanguíneo obtuvo mejoras más importantes sobre el diámetro del brazo, el área de sección transversal y la fuerza dinámica máxima en un ejercicio de extensión de brazos. Las sensaciones prominentes reportadas en el brazo en que se utilizó el torniquete fueron presión y dolor (Wheatherholt y col., 2013).

- Manimmanakorn y col. (2013) quisieron comprobar las posibles diferencias producidas entre un entrenamiento de fuerza con restricción del flujo sanguíneo con respecto a un grupo de entrenamiento de fuerza ante estímulos de hipoxia intermitente (IHT). Los sujetos de dicho estudio, mujeres con experiencia en el entrenamiento de fuerza, realizaron durante: 5 semanas, con 3 sesiones consistentes en 3 series al fallo con una carga relativa del $20 \%$ de 1 RM en ejercicios de flexión y extensión de piernas. Se usó una presión oclusiva de aproximadamente $230 \mathrm{mmHg}$, y aire hipóxico para generar una saturación de oxígeno en la sangre arterial de $\sim 80 \%$. Los resultados evidenciaron mejoras en los niveles de fuerza (entre el 11 y 15\%) y en el área de sección transversal de los cuádriceps que, aunque son igualmente significativas, fueron ligeramente superiores en el grupo de entrenamiento con restricción del flujo sanguíneo $(7,6 \%)$ con respecto al grupo de entrenamiento con IHT (5,3\%).

- Este estudio tuvo como objetivo conocer las adaptaciones de la fuerza muscular y el grosor del músculo esquelético después de dos volúmenes diferentes de entrenamiento de restricción del flujo sanguíneo (BFRT) y compararlos con el entrenamiento de alta intensidad. La muestra se dividió en cuatro grupos: BFRT de bajo volumen y baja intensidad (BFRT LV); BFRT de alto volumen y baja intensidad (BFRT HV); entrenamiento de resistencia tradicional de alta intensidad (HIT); y un grupo de control, que mantuvo sus actividades rutinarias (CON). La extensión de la pierna a una repetición 
máxima (1 RM), así como el grosor muscular del recto femoral (RF) y vastus lateralis (VL) se evaluaron al inicio $y$ después de 5 semanas de entrenamiento. Aumentaron el rendimiento de $1 \mathrm{RM}$ los grupos BFRT LV (7.03\%), BFRT HV (6.24\%,) y HIT (18.86\%), mientras que no se observaron cambios en el grupo CON. El grosor muscular de RF y VL se incrementó independientemente del grupo de entrenamiento $\quad(7.5 \%, \quad$ y $9.9 \%$, respectivamente). Los autores llegan a la conclusión de que duplicar el volumen de ejercicio con el entrenamiento de restricción del flujo sanguíneo, no produce ningún beneficio adicional con el tamaño muscular o la fuerza. Aunque se observaron aumentos similares en el grosor muscular entre los grupos, el entrenamiento de resistencia tradicional de alta intensidad aumentó el rendimiento de 1 RM en mayor medida en comparación con cualquiera de los volúmenes de BFRT. (Martín, Marín, Menéndez, Ferrero, Loenneke, y Herrero 2013).

- Un estudio de Abe et al. (2005) examinaron el cambio diario en la fuerza muscular y el tamaño muscular durante 7 días de entrenamiento de restricción del flujo sanguíneo vascular. La atención se centró en un sujeto, 27 años y un hombre con experiencia en entrenamiento de resistencia, pero el sujeto no entrenó en los tres meses anteriores. $\mathrm{El}$ entrenamiento de resistencia de baja intensidad con restricción de flujo sanguíneo en los músculos de las piernas se realizó dos veces al día durante 7 días consecutivos. El volumen de entrenamiento y la carga realizaban 3 series de 15 repeticiones con 30 segundos de descanso.
La intensidad se realizó al 20\% de 1-RM. Las presiones utilizadas durante la restricción del flujo sanguíneo oscilaron entre 160 y 220 $\mathrm{mmHg}$, comenzando con $160 \mathrm{mmHg}$ en el primer día, aumentando en $20 \mathrm{mmHg}$ cada día. La imagen muscular completa se realizó mediante una MRI en el músculo cuádriceps y la fuerza isométrica absoluta se midió con un dinamómetro isocinético. Después de una semana, el sujeto ganó fuerza absoluta (303 $\mathrm{Nm}$ desde la línea de base de $257 \mathrm{Nm}$ ) y aumentó el tamaño muscular (3.5\% desde la línea de base) después de una semana de entrenamiento de restricción del flujo sanguíneo vascular de baja intensidad. Se ha demostrado que los resultados similares encontrados por este estudio ocurren en 8 a 12 semanas de entrenamiento.

\section{Aportes}

Se encuentran aportes significativos que Ilenan y enriquecen no solo al trabajo escrito, sino también, para aquellos que realizaron la búsqueda de la información y para aquellos lectores que comprenden un tema con mucho más a detalle. Para el caso en concreto, los mayores aportes de los artículos son:

- Métodos de entrenamiento con restricción del flujo sanguíneo, en que encontramos el volumen, las repeticiones, series, intensidad del entrenamiento y cargas que, según varios autores de diferentes artículos, encontraban resultados con un aumento de la masa muscular y un aumento en la fuerza máxima con este método. (Schoenfeld, J, 2012), (Yasuda, Loenneke, Thiebaud, \& Abe, 2015), (Yasuda, Loenneke, Thiebaud, \& Abe, 2012), (Madarame, Neya, Ochi, Nakazato, Sato, \& Ishii, 2008), (Hernández, Azael, 
2012), (Hernández, Marín, y Herrero, 2011).

- Este método de entrenamiento no solo se usa en halterofilia o en el fitness, entrenadores de deportes como el fútbol lo adoptaron como nueva metodología de entrenamiento, para así, acelerar procesos de adaptación a la fuerza máxima. (Manimmanakorn, Manimmanakorn, Taylor, Draper, Billaut, Shearman, \& Hamlin, 2013), (Yamanaka, Farley, \& Caputo, 2013).

- Este entrenamiento hace un reclutamiento preferente de las fibras de contracción rápida, estas fibras se reclutan normalmente sólo con los esfuerzos de alta intensidad, aproximadamente del 70\% del 1 RM. (Cook, Clark, and Ploutz-Snyder, 2007), (Sumide, Sakuraba, Sawaki, Ohmura and Tamura 2009), (Wolinski, Neves, \& Pietrovski, 2013), (O'Halloran, 2014), (Behi, Amani, Fahey, \& Afsharnezhad, 2017).

- A pesar que el entrenamiento con restricción de flujo sanguíneo se realiza de forma local, se ve también beneficiados otros músculos cercanos, experimentando un desarrollo. (Laurentino, Ugrinowitsch, Aihara, Fernandes, Parcell, Ricard, et al, 2008), (Patterson, Ferguson, 2010), (Kubo, Komuro, Ishiguro, Tsunoda, Sato \& Ishii, et al., 2006), (Shinohara, Kouzaki. Yoshihisa, Fukunaga, 1998).

- Al entrenar con dicho método tiene también un efecto cruzado, es decir, que, al entrenar una extremidad, la extremidad opuesta, también obtiene dicho beneficio (Wilkinson, y Cols., 2006).

También, se encuentran artículos referentes a los riesgos que este método podría causar a partir de dicho método, sobre todo en el área de las tuberculosis, generada por una posible coagulación de la sangre.
En general, también, hay contenido en donde estas investigaciones utilizan determinadas rutinas usadas en ciertos grupos de control en un plazo entre 4 semanas hasta 16 semanas. para visualizar las ganancias, las cuales se expondrán mucho más adelante con mayor detalle.

Por la variedad de los artículos encontrados enfocados hacia la hipertrofia y la fuerza muscular, es adecuado realizar una categorización, que sea de ayuda para que el lector pueda visualizar de manera clara, cuantos artículos se han escrito y hacia qué tema se han centrado, siendo así, de gran ayuda para aquellos que quieran tener ideas para poder realizar determinadas investigaciones en este campo, conozcan que temas pueden centrarse con mayor facilidad por el poco contenido.

El entrenamiento de restricción de flujo sanguíneo también podría aplicarse en astronautas para prevenir la atrofia muscular cuando estos se encuentren en gravedad cero. (Nakakima, 2008). Sin embargo, los efectos de este método aún se desconocen en la micro gravedad. Se realizó un estudio en el que se aplicaba la restricción de flujo sanguíneo para observar las respuestas hemodinámicas durante la ingravidez simulada mientras el paciente se encontraba de pie y sentado. Concluyeron que reproduce un estrés similar a estar en la gravedad durante la ingravidez simulada, lo que generaría un estímulo adecuado para evitar la atrofia muscular en astronautas (Nakajima, lida, Kurano, Takano, Morita, Meguro, Abe, 2008). Conservar la masa muscular es fundamental ya que se conserva la movilidad y la fuerza del astronauta cuando este se encuentra en gravedad cero, lo que beneficiaría significativamente el regreso a la superficie terrestre. 
Hay que resaltar el estudio que realizaron Abe y colaboradores (2006), en el que se llevó a cabo una serie de pautas con el entrenamiento de restricción de flujo sanguíneo a un total de 19 hombres y mujeres activos, de entre 60 y 78 años. Se aleatorizaron en un grupo de entrenamiento de caminata KAATSU ( $\mathrm{n}=11$, K-walk) o un grupo de control sin ejercicios ( $n=8$, control). El grupo K-walk realizó caminatas en cinta de 20 minutos ( $67 \mathrm{~m} / \mathrm{min}$ ), 5 días / semana durante 6 semanas, con una presión de 50 $\mathrm{mmHg}$. La capacidad funcional aumentó significativamente solo en el grupo K-walk, sin embargo, no hubo cambios en el consumo máximo de oxígeno estimado (absoluto y relativo a la masa corporal) para ninguno de los grupos.

Los resultados del estudio actual indican que 6 semanas de entrenamiento KAATSUwalk no mejoraron simultáneamente la capacidad cardiovascular y muscular de los participantes mayores. Sin embargo, aumentó significativamente el tamaño y la fuerza muscular, así como la capacidad funcional de hombres y mujeres mayores activos (Abe, Sakamaki, Fujita, Ozaki, Sugaya, Sato, Yoshiaki, \& Nakajima, Toshiaki, 2010). Esto indica que el principal efecto con el método de restricción de flujo sanguíneo es la hipertrofia muscular, esta a su vez, trae consigo la fuerza, sin embargo, no mejora la resistencia aeróbica directamente.

\section{MARCO TEÓRICO}

Las mayores evidencias físicas visuales que se pueden apreciar a nivel externo es la fuerza y el aumento de la masa muscular (Pope, Willardson \& Schoenfeld, 2013). Sin embargo, en el interior del organismo a nivel metabólico, endocrino, y morfológico, suceden procesos diferentes a los presentados normalmente por entrenamientos tradicionales.

A nivel endocrino se puede apreciar que sí, hay un aumento continuo, en los niveles de lactato en sangre, esto haría que se aumentaran los niveles de la hormona de crecimiento, mejor conocida por sus siglas $\mathrm{GH}$ (Takarada y col., 2000). Estos niveles son superiores a los reflejados en un entrenamiento con cargas tradicionales que oscilan entre el $70 \%$ y el $85 \%$ del 1 RM (Reeves, Kraemer, Hollander, Clavier, Thomas, Francois, y Castracane, Sato, Yoshitomi, y Abe, 2006). Este mismo aumento de la hormona de crecimiento induce el aumento progresivo del músculo.

A nivel metabólico, se ha demostrado que el entrenamiento oclusivo puede aumentar los niveles de hormona de crecimiento hasta un $290 \%$ en comparación con el entrenamiento tradicional.

sin embargo, aún no está claro cómo se relaciona esto con la hipertrofia muscular. (Takarada, Nakamura, Aruga, Onda, Miyazaki, Ishii, 2000). También aumenta la síntesis de proteínas musculares, la señalización de mTOR (una enzima responsable de la síntesis de proteínas musculares), una mayor activación de las células satélite (otro proceso que influye en la hipertrofia) y disminuye los niveles de miostatina (un tipo de proteína que limita el crecimiento muscular). (Mohamed, Basant, y Larion, 2017).

Otro de las posibles explicaciones sobre el sorprendente efecto anabólico al entrenar usando torniquetes para la restricción parcial del flujo sanguíneo, es por la reducción de la hormona cortisol, debido a que esta hormona presenta un efecto catabólico en el ejercicio, es decir, una degradación parcial de las proteínas y aminoácidos que se encuentran en los músculos (Abe y col., 2005), también, 
reduciendo al máximo la creatina quinasa, porque, sí, se encuentran altos niveles en una muestra de sangre indica generalmente que el músculo está siendo degradado, ya sea por una patología o condiciones, como la fiebre o el esfuerzo muscular (Thiebaud, Yasuda, Loenneke, y Abe, 2013).

Todos estos factores anti catabólicos se integran para crear un ambiente favorable en el aumento de fuerza y desarrollo de masa muscular magra.

Fuera de todo lo beneficioso que parezca, aún faltan investigaciones sobre los riesgos, consecuencias o efectos secundarios, sin embargo, una investigación evaluó la aparición de trombos realizando el entrenamiento con restricción del flujo sanguíneo (Madarame, Kurano, Takano, lida, Sato, Ohshima, Abe, Ishii, Morita, y Nakajima, 2010).

En esta investigación se trabajaron 4 series en la prensa, para las piernas al 30\% del 1RM. No se observaron coágulos en formación que provoquen trombos. En dicha investigación, tras 4 series de prensa de piernas al 30\% de 1 RM no se observaron incrementos en los niveles de protombina $1+20$ antitrombina 3 compleja (son proteínas que ayudan a coagulación de la sangre), ni degradación de la fibrina (proteína que actúa como pegamento cuando se expone una herida). Por tanto, el entrenamiento con restricción del flujo sanguíneo no pareció tener influencia en la formación de trombos (Madarame y col., 2010).

Por otro lado, la investigación existente sobre este entrenamiento, que se confirma en un alto porcentaje de seguridad al realizarlo, hay que tener en cuenta, que estas investigaciones se han llevado acabo con población aparentemente sana (Pope, Willardson, y Schoenfeld, 2013). Esto lleva a recomendar a aquellas personas que tienen: patologías cardiovasculares, hipertensión, diabetes, pacientes renales, deben abstenerse a la realización de dicho método.

La investigación ha demostrado que la oclusión vascular moderada causa numerosas adaptaciones fisiológicas positivas con cargas tan bajas como $10-30 \%$ de la capacidad máxima de trabajo. Típicamente, de 3 a 5 series a la fatiga volitiva (voluntad de dar más repeticiones) con un descanso de 30 segundos a 1 minuto entre series (Loenneke, \& Pujol, Joseoh, 2009). La oclusión se puede aplicar con un aparato KAATSU o más prácticamente mediante rodilleras elásticas. La presión solo necesita ser lo suficientemente alta como para bloquear el retorno venoso ( 50-100 mm Hg) (Sumide, T., Sakuraba K., Sawaki, K., Ohmura, $\mathrm{H}$., and Tamura, $\mathrm{Y}, 2009$ ). El entrenamiento de oclusión se puede aplicar a los atletas; pacientes en rehabilitación postoperatoria, específicamente lesiones del LCA; pacientes con rehabilitación cardíaca; los ancianos; e incluso astronautas para combatir la atrofia y cuando se aplica con ejercicio para inducir hipertrofia muscular.

\section{DISEÑO METODOLÓGICO}

\section{Tipo de estudio}

Este trabajo se denomina revisión bibliográfica debido a que tiene como fin inspeccionar las investigaciones publicadas y situarlas en cierta perspectiva, es decir, recopilar información ya existente sobre un tema, proporciona así, una visión sobre el estado problema elegido a tratar. (Alfonso, 2017).

Específicamente las búsqueda de información del tema es acerca del 
entrenamiento con restricción de flujo sanguíneo.

\section{Enfoque}

En esta investigación se presenta un enfoque cualitativo, debido a que se recopila información de artículos en las diferentes bases de datos y los resultados se darán a conocer de manera analítica, junto con información que va acompañada de la cantidad de investigaciones que se encontraron en total, los funcionales, y la propuesta metodológica, donde se describe en porcentaje la carga y de manera numérica las series y repeticiones, junto con la frecuencia de entrenamiento. La aproximación cualitativa evalúa el desarrollo natural de los sucesos, es decir, no hay manipulación ni estimulación de la realidad. (Guajardo, \& Castro, 2007).

\section{Diseño}

Se presenta un diseño de forma transversal (este recopila datos en un sólo momento, en un tiempo único). Siendo este diseño pertinente a esta investigación, porque como se menciona anteriormente, se ejecutará un estudio de revisión (Reyes, 2013).

\section{Alcance investigativo}

El presente trabajo se determina que es descriptivo, debido a que se describe el proceso de entrenamiento de restricción de flujo sanguíneo, junto con las variables de entrenamiento (intensidad, densidad, volumen, frecuencia), y a nivel fisiológico, se describe la respuesta metabólica, endocrina, morfológica luego de haber usado dicho método. (Hernández, Fernández y Batista, 2010)

\section{Criterios de inclusión}

La Fecha: Esta no debe superar los 10 años (excepto artículos donde se sustenta conceptos o términos generales)

Bases de datos: Se eligieron las bases de datos más conocidas y que se encuentran en la universidad de ciencias aplicadas y ambientales U.D.C.A. a disposición de los estudiantes.

Tema: Se tomará en cuenta que los artículos tengan como tema principal el entrenamiento con restricción de flujo sanguíneo junto con la hipertrofia muscular.

\section{Criterios de exclusión}

Todos aquellos elementos que no cumplan con los parámetros mencionados en el punto anterior.

\section{Materiales, Métodos e instrumentos de recolección de datos}

Consiste en una revisión bibliográfica en las bases de datos, donde se limitó el año de publicación de los artículos, tomando un lapso de 10 años como adecuado, es decir, entre el año de 2008 a 2018. La mayor parte de los artículos están escritos en inglés, como idiomas principales.

Para algunas definiciones claves de la revisión, no se estableció un límite de año, porque, a pesar del tiempo, muchos conceptos siguen vigentes, como conceptos 
de hipertrofia, de fuerza, de investigación, tipos de alcance, entre otros más.

La técnica para la recopilación de los datos se le denomina, investigación documental, debido este instrumento constituye un apoyo inmediato, no implica ningún costo y es fácilmente cuantificable.
El medio para la búsqueda de información fue mediante cinco (5) bases de datos, las cuales son: ProQuest, ScienceDirect, SportDiscus, Scopus y PubMed.

La búsqueda se realizó en las bases de ProQuest (12), ScienceDirect (8), SportDiscus (15), Scopus (12) y PubMed (13).

\section{Matriz Operatividad de variables}

OBJETIVO GENERAL: Describir los procesos de hipertrofia y de fuerza mediante el uso del entrenamiento con restricción de flujo sanguíneo.

\begin{tabular}{|c|c|c|c|c|}
\hline OBJETIVO & VARIABLE & DIMENSIÓN & INDICADOR & INSTRUMENTO \\
\hline $\begin{array}{l}\text { Revisar escritos } \\
\text { existentes en el que se } \\
\text { describe acerca del } \\
\text { entrenamiento con } \\
\text { restricción de flujo } \\
\text { sanguíneo. }\end{array}$ & $\begin{array}{l}\text { Escritos sobre } \\
\text { entrenamiento con } \\
\text { restricción de flujo } \\
\text { sanguíneo. }\end{array}$ & $\begin{array}{l}\text { Artículos de } \\
\text { investigación. }\end{array}$ & $\begin{array}{l}\text { Publicación } \\
\text { académica }\end{array}$ & $\begin{array}{c}\text { Base de datos } \\
\text { ProQuest, } \\
\text { ScienceDirect, } \\
\text { SportDiscus, Scopus } \\
\text { y PubMed }\end{array}$ \\
\hline $\begin{array}{l}\text { Determinar los } \\
\text { beneficios y del } \\
\text { entrenamiento con } \\
\text { restricción de flujo } \\
\text { sanguíneo. }\end{array}$ & $\begin{array}{c}\text { Beneficios } \\
\text { fisiológicos en } \\
\text { cuanto a sistema } \\
\text { muscular. }\end{array}$ & Tipos de beneficios. & $\begin{array}{c}\text { Fibras } \\
\text { involucradas } \\
\text { Hormonas } \\
\text { segregadas } \\
\text { Daño muscular }\end{array}$ & $\begin{array}{c}\text { Base de datos } \\
\text { ProQuest, } \\
\text { ScienceDirect, } \\
\text { SportDiscus, Scopus } \\
\text { y PubMed }\end{array}$ \\
\hline $\begin{array}{l}\text { Proponer una estructura } \\
\text { metodológica de la } \\
\text { oclusión para la } \\
\text { hipertrofia. }\end{array}$ & $\begin{array}{c}\text { Estructura } \\
\text { metodológica de } \\
\text { entrenamiento. }\end{array}$ & $\begin{array}{c}\text { Carga de } \\
\text { entrenamiento. }\end{array}$ & $\begin{array}{l}\text { Frecuencia. } \\
\text { Intensidad. } \\
\text { Volumen. } \\
\text { Densidad. }\end{array}$ & $\begin{array}{c}\text { Base de datos } \\
\text { ProQuest, } \\
\text { ScienceDirect, } \\
\text { SportDiscus, Scopus } \\
\text { y PubMed }\end{array}$ \\
\hline
\end{tabular}

Tabla 3 Matriz de variables Fuente: Elaboración propia 


\section{RESULTADOS}

La siguiente tabla hace referencia a la revisión documental de los principales artículos.

\begin{tabular}{|c|c|c|c|c|}
\hline AUTOR & TÍTULO & OBJETIVO & MÉTODO & APORTES \\
\hline $\begin{array}{c}\text { Yamanaka, Farley y } \\
\text { Caputo, (2013) }\end{array}$ & $\begin{array}{l}\text { El entrenamiento de } \\
\text { oclusión aumenta la } \\
\text { fuerza muscular en los } \\
\text { jugadores de fútbol de } \\
\text { división IA. }\end{array}$ & $\begin{array}{l}\text { Investigar la efectividad } \\
\text { de } 4 \text { semanas de } \\
\text { entrenamiento de } \\
\text { resistencia de baja } \\
\text { intensidad con oclusión } \\
\text { del flujo sanguíneo en } \\
\text { la hipertrofia muscular } \\
\text { de la parte superior e } \\
\text { inferior y la fuerza } \\
\text { muscular en jugadores } \\
\text { de fútbol. }\end{array}$ & $\begin{array}{c}4 \text { series de press de } \\
\text { banca y sentadilla. } 30 \\
\text { repeticiones de } 20 \% \text { de } \\
1 \mathrm{RM}, 3 \text { veces por } \\
\text { semana, durante } 4 \\
\text { semanas. }\end{array}$ & $\begin{array}{c}\text { Demostraron aumentos } \\
\text { del } 1 \text { RM en press de } \\
\text { banca y en sentadillas } \\
\text { ( } 7,0 \text { y } 8,0 \%, \\
\text { respectivamente), y } \\
\text { aumentos en las } \\
\text { circunferencias del } \\
\text { tórax superior e inferior } \\
\text { ( } 3 \text { y } 3 \%, \\
\text { respectivamente). }\end{array}$ \\
\hline $\begin{array}{l}\text { Yasuda, Loenneke, } \\
\text { Thiebaud y Abe (2012) }\end{array}$ & $\begin{array}{c}\text { Los efectos del flujo } \\
\text { sanguíneo restringido } \\
\text { en el entrenamiento } \\
\text { concéntrico o } \\
\text { excéntrico de baja } \\
\text { intensidad sobre el } \\
\text { tamaño y la fuerza } \\
\text { muscular. }\end{array}$ & $\begin{array}{l}\text { Investigaron los } \\
\text { efectos agudos y } \\
\text { crónicos de manera } \\
\text { concéntrica y } \\
\text { excéntrica usando el } \\
\text { entrenamiento de } \\
\text { restricción de flujo } \\
\text { sanguíneo con baja } \\
\text { intensidad, sobre el } \\
\text { tamaño y la fuerza } \\
\text { muscular. }\end{array}$ & $\begin{array}{l}\text { Realizaron flexión de } \\
\text { codo con mancuernas } \\
\text { (curl de bíceps) con } \\
\text { una carga del } 30 \% \text { del } \\
1 \mathrm{RM} \text {, con cuatro } \\
\text { series de un total de } 75 \\
\text { repeticiones, } 3 \text { dias por } \\
\text { semana durante } 6 \\
\text { semanas. Un brazo de } \\
\text { manera concéntrica y } \\
\text { el otro excéntrica. }\end{array}$ & $\begin{array}{l}\text { se observaron mejoras } \\
\text { significativamente } \\
\text { superiores, en los } \\
\text { valores de fuerza } \\
\text { máxima isométrica } \\
(8,6 \%) \text { medido por } \\
\text { electromiografía, y el } \\
\text { área de sección } \\
\text { transversal del brazo } \\
(12 \%) \text { y del volumen } \\
\text { muscular (11,7\%) en el } \\
\text { brazo que se ejercitó } \\
\text { concéntricamente con } \\
\text { respecto al que se } \\
\text { ejercitó } \\
\text { excéntricamente. }\end{array}$ \\
\hline $\begin{array}{l}\text { Weatherholt, Beekley, } \\
\text { Greer, Urtel y Mikesky } \\
\text { (2013) }\end{array}$ & $\begin{array}{l}\text { Formación modificada } \\
\text { de Kaatsu: } \\
\text { adaptaciones y } \\
\text { percepciones de los } \\
\text { sujetos }\end{array}$ & $\begin{array}{l}\text { investigaron las } \\
\text { adaptaciones } \\
\text { musculares a } 40 \\
\text { sujetos jóvenes } \\
\text { después de realizar un } \\
\text { entrenamiento } \\
\text { oclusivo. }\end{array}$ & $\begin{array}{l}\text { durante } 8 \text { semanas, } 3 \\
\text { veces por semana } \\
\text { realiza } 3 \text { series de } 15 \\
\text { repeticiones de } \\
\text { ejercicios de bíceps y } \\
\text { tríceps al } 20 \% \text { el } 1 \mathrm{RM} \\
\text { para un grupo, el otro } \\
\text { grupo realizaba el } \\
\text { mismo trabajo sin } \\
\text { torniquete. }\end{array}$ & $\begin{array}{l}\text { Se comprobó que los } \\
\text { brazos que se } \\
\text { ejercitaron con } \\
\text { restricción del flujo } \\
\text { sanguíneo obtuvo } \\
\text { mejoras más } \\
\text { importantes sobre el } \\
\text { diámetro del brazo, el } \\
\text { área de sección } \\
\text { transversal y la fuerza } \\
\text { dinámica máxima en } \\
\text { un ejercicio de } \\
\text { extensión de brazos. }\end{array}$ \\
\hline
\end{tabular}




\begin{tabular}{|c|c|c|c|c|}
\hline $\begin{array}{l}\text { Manimmanakorn, } \\
\text { Manimmanakorn, } \\
\text { Taylor, Draper, } \\
\text { Billaut, Shearman y } \\
\text { Hamlin, (2013) }\end{array}$ & $\begin{array}{c}\text { Efectos del } \\
\text { entrenamiento de } \\
\text { resistencia combinado } \\
\text { con oclusión vascular o } \\
\text { hipoxia en la función } \\
\text { neuromuscular en } \\
\text { atletas }\end{array}$ & $\begin{array}{l}\text { comprobar las posibles } \\
\text { diferencias producidas } \\
\text { entre un entrenamiento } \\
\text { de fuerza con } \\
\text { restricción del flujo } \\
\text { sanguíneo con } \\
\text { respecto a un grupo de } \\
\text { entrenamiento de } \\
\text { fuerza ante estímulos } \\
\text { de hipoxia intermitente }\end{array}$ & $\begin{array}{c}5 \text { semanas, con } 3 \\
\text { sesiones consistentes } \\
\text { en } 3 \text { series al fallo, } \\
\text { carga relativa del } 20 \% \\
\text { de } 1 \mathrm{RM} \text {, ejercicios de } \\
\text { flexión y extensión de } \\
\text { piernas. Se usó una } \\
\text { presión de } 230 \mathrm{mmHg} \\
\text { y aire hipóxico para } \\
\text { generar una saturación } \\
\text { de oxígeno en la } \\
\text { sangre arterial de } \\
80 \% \text {. }\end{array}$ & $\begin{array}{l}\text { mejoras en los niveles } \\
\text { de fuerza (entre el } 11 \text { y } \\
15 \% \text { ) y en el área de } \\
\text { sección transversal de } \\
\text { los cuádriceps que, } \\
\text { aunque son igualmente } \\
\text { significativas, fueron } \\
\text { ligeramente superiores } \\
\text { en el grupo de } \\
\text { entrenamiento con } \\
\text { restricción del flujo } \\
\text { sanguíneo }(7,6 \%)\end{array}$ \\
\hline $\begin{array}{c}\text { Martín, Marín, } \\
\text { Menéndez, Ferrero, } \\
\text { Loenneke, y Herrero } \\
2013\end{array}$ & $\begin{array}{c}\text { Adaptaciones } \\
\text { musculares después } \\
\text { de dos volúmenes } \\
\text { diferentes de } \\
\text { entrenamiento de } \\
\text { restricción del flujo } \\
\text { sanguíneo }\end{array}$ & $\begin{array}{c}\text { conocer las } \\
\text { adaptaciones de la } \\
\text { fuerza muscular y el } \\
\text { grosor del músculo } \\
\text { esquelético después } \\
\text { de dos volúmenes } \\
\text { diferentes de } \\
\text { entrenamiento de } \\
\text { restricción del flujo } \\
\text { sanguíneo }\end{array}$ & $\begin{array}{c}5 \text { semanas, } 2 \\
\text { sesiones, grupo 1: } 3 \text { x } \\
1520 \% \text { RM en } \\
\text { extensión de pierna. R } \\
\text { de } 1 \text { min. Grupo } 2: 2 \text { ( } 3 \\
\text { x 15) } 20 \% \text { RM en } \\
\text { extensión de pierna. R: } \\
1 \text { y } 5 \text { min. Grupo 3: } 3 \text { x } \\
8 \text { al } 85 \% \text { RM en } \\
\text { extensión de pierna. R } \\
1 \text { min }\end{array}$ & $\begin{array}{l}\text { Duplicar el volumen de } \\
\text { ejercicio con el } \\
\text { entrenamiento de } \\
\text { restricción del flujo } \\
\text { sanguíneo, no produce } \\
\text { ningún beneficio } \\
\text { adicional con el } \\
\text { tamaño muscular o la } \\
\text { fuerza. el } \\
\text { entrenamiento de } \\
\text { tradicional de alta } \\
\text { intensidad aumentó el } \\
\text { rendimiento de 1 RM } \\
\text { en comparación con } \\
\text { cualquiera de los } \\
\text { volúmenes de BFRT. }\end{array}$ \\
\hline $\begin{array}{l}\text { Abe, Charles, Kearns y } \\
\text { Sato, (2005) }\end{array}$ & $\begin{array}{l}\text { El cambio diario en la } \\
\text { fuerza muscular y el } \\
\text { tamaño del músculo } \\
\text { esquelético durante } 7 \\
\text { días. Entrenamiento de } \\
\text { resistencia con } \\
\text { KAATSU: Un estudio } \\
\text { de caso. }\end{array}$ & $\begin{array}{l}\text { el cambio diario en la } \\
\text { fuerza muscular y el } \\
\text { tamaño muscular } \\
\text { durante } 7 \text { días de } \\
\text { entrenamiento de } \\
\text { restricción del flujo } \\
\text { sanguíneo vascular. }\end{array}$ & $\begin{array}{l}\text { Realizó } 3 \text { series de } 15 \\
\text { repeticiones con } 30 \\
\text { segundos de } \\
\text { descanso. La } \\
\text { intensidad se realizó al } \\
20 \% \text { de } 1-\mathrm{RM} \text {. Las } \\
\text { presiones utilizadas } \\
\text { durante la restricción } \\
\text { del flujo sanguíneo } \\
\text { oscilaron entre } 160 \text { y } \\
220 \mathrm{mmHg}\end{array}$ & $\begin{array}{l}\text { El sujeto ganó fuerza } \\
\text { absoluta ( } 303 \mathrm{Nm} \\
\text { desde la línea de base } \\
\text { de } 257 \mathrm{Nm} \text { ) y aumentó } \\
\text { el tamaño muscular } \\
\text { (3.5\% desde la línea } \\
\text { de base) }\end{array}$ \\
\hline $\begin{array}{l}\text { Wilkinson, S., y Cols. } \\
(2006)\end{array}$ & $\begin{array}{c}\text { La hipertrofia con } \\
\text { ejercicio de resistencia } \\
\text { unilateral se produce } \\
\text { sin aumentos en la } \\
\text { concentración de } \\
\text { hormonas anabólicas } \\
\text { endógenas }\end{array}$ & $\begin{array}{l}\text { Comprender mejor el } \\
\text { papel que desempeñan } \\
\text { los aumentos } \\
\text { transitorios de las } \\
\text { hormonas anabólicas } \\
\text { en la hipertrofia } \\
\text { muscular con el } \\
\text { entrenamiento de } \\
\text { resistencia unilateral. }\end{array}$ & $\begin{array}{l}\text { Diez sujetos varones } \\
\text { jóvenes realizaron } \\
\text { entrenamiento de } \\
\text { resistencia unilateral } \\
\text { durante } 8 \text { semanas ( } 3 \\
\text { días / semana). }\end{array}$ & $\begin{array}{l}\text { Al entrenar con dicho } \\
\text { método tiene también } \\
\text { un efecto cruzado, es } \\
\text { decir, que, al entrenar } \\
\text { una extremidad, la } \\
\text { extremidad opuesta, } \\
\text { también obtiene dicho } \\
\text { beneficio }\end{array}$ \\
\hline
\end{tabular}


Laurentino, G., Ugrinowitsch, C, Aihara, Y., Fernandes, R., Parcell, C., Ricard, M., et al (2008)
Efectos del entrenamiento de la fuerza y la oclusión vascular. determinar si la oclusión vascular produjo un efecto aditivo en la hipertrofia muscular y el rendimiento de la fuerza con cargas de entrenamiento de alta fuerza
Dieciséis hombres, dos grupos: entrenamiento de alta intensidad $(\mathrm{HI}=$ $6 \mathrm{RM}$ ) y entrenamiento de intensidad moderada $(\mathrm{Ml}=12$ $\mathrm{RM})$. Se colocó un manguito de oclusión en el extremo proximal del muslo derecho, La pierna izquierda sirvió como control, por lo que se entrenó sin oclusión vascular. Quince hombres sin entrenamiento, dos grupos, (flexión de brazo) al $50 \%$ del máximo de una repetición (1RM).

Después del ejercicio del brazo, el OCC realizó el ejercicio de la pierna con restricción del flujo sanguíneo (30\% de 1RM, tres series, 15-30

repeticiones), mientras que NOR realizó el mismo ejercicio de la pierna sin oclusión. La sesión de entrenamiento se realizó dos veces por semana durante 10 semanas.

Los ejercicios incluían correr distancias de 20 y 40 metros. Los sujetos tomaron un descanso de 20 segundos después de cada repetición y 2 minutos de descanso entre series. el entrenamiento de resistencia de baja intensidad aumenta el tamaño muscular y la fuerza cuando se combina con ejercicios de resistencia con restricción del flujo sanguíneo para otros grupos musculares. Se sugirió que cualquier factor (s) circulante estaba involucrado en este efecto remoto del ejercicio sobre el tamaño muscular.
Behi, Amani, Fahey y Afsharnezhad, (2017). entrenamiento de intervalos de alta intensidad con restricción de sangre en el rendimiento anaeróbico

\section{rendimiento anaeróbico} entre los atletas jóvenes. efecto de los entrenamientos HIIT Kaatsu en el

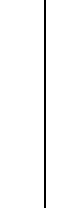

La oclusión vascular en combinación con el fuerza de alta intensidad no aumenta comparación con el entrenamiento de fuerza de alta intensidad solo.

Tabla 4 Resultados

Referencia: Elaboración propia 
Para una mejor comprensión para el lector acerca de los artículos que se han analizado, se les ha asignado categorías según la finalidad del estudio en particular. Estas categorías son acordes al tema central de los artículos.

A continuación, se encuentra el listado de los artículos con su respectiva categorización:

\section{Adaptaciones}

1. Respuestas y adaptaciones al entrenamiento de fuerza oclusivo de baja intensidad. (Hernández, y Azael, 2012).

2. Aumento en el flujo sanguíneo postoclusivo de la pantorrilla y la fuerza después del entrenamiento de resistencia al ejercicio a corto plazo con restricción del flujo sanguíneo en mujeres jóvenes. (Patterson, y Ferguson, 2010).

3. El tamaño y la fuerza muscular se incrementan después del entrenamiento a pie con flujo sanguíneo venoso restringido desde el músculo de la pierna, entrenamiento de Kaatsu-walk. (Abe, Kearns, y Sato, 2006).

4. Adaptaciones y percepciones del sujeto. (Weatherholt, Beekley, Greer, Urtel, \& Mikesky, 2013).

5. Las adaptaciones musculares después de dos volúmenes diferentes de flujo sanguíneo restringieron el entrenamiento. (Martín-Hernández, Marín, Menéndez, Ferrero, Loenneke, y Herrero, 2013),

6. Un entrenamiento con resultados más grandes. (Warnock, y Bachman, 2015).

7. Presión de oclusión del flujo sanguíneo en reposo e inmediatamente después de una sesión de ejercicio de carga baja. (Barnett, Dankel, Counts, Nooe, Abe, Loenneke, 2016).
8. Adaptaciones neuromusculares en el músculo humano después del entrenamiento de resistencia de baja intensidad con oclusión vascular. (Moore, Burgomaster, Schofield, Gibala, Saie, y Phillips, 2004).

9. Entrenamiento de fuerza con oclusión vascular posibles mecanismos adaptativos. (De Castro, Aquino, Berti, Gonçalves, y Puggina, 2017).

\section{Efectos}

1. Efectos del desentrenamiento después del entrenamiento concéntrico o excéntrico de baja intensidad restringido al flujo sanguíneo sobre el tamaño y la fuerza muscular. (Yasuda, Loenneke, Thiebaud, y Abe, 2015).

2. Efectos del entrenamiento sanguíneo restringido de baja intensidad concéntrica o excéntrico sobre el tamaño y la fuerza muscular. (Yasuda, Loenneke, Thiebaud, \& Abe, 2012).

3. Efectos de transferencia del entrenamiento de resistencia con restricción de flujo sanguíneo. (Madarame, Neya, Ochi, Nakazato, Sato, \& Ishii, 2008).

4. Efectos del entrenamiento de fuerza y la oclusión vascular. (Laurentino, Ugrinowitsch, Aihara, Fernandes, Parcell, Ricard, et al., 2008).

5. Efectos del entrenamiento de resistencia a baja carga con la oclusión vascular sobre las propiedades mecánicas del músculo y el tendón. (Kubo, Komuro, Ishiguro, Tsunoda, Sato \& Ishii, et al., 2006).

6. Efectos del entrenamiento de resistencia combinado con vascular oclusión o hipoxia en la función neuromuscular en atletas.

(Manimmanakorn, 
Manimmanakorn, Taylor, Draper, Billaut, Shearman, \& Hamlin, 2013).

7. Efecto del entrenamiento con ejercicios de resistencia combinado con una oclusión vascular relativamente baja. (Sumide, Sakuraba, Ohmura, y Tamura, 2009).

8. Efecto del entrenamiento de Kaatsu sobre la electroforesis de proteínas séricas y el nivel de rendimiento de 100 metros Obstáculos para estudiantes universitarias. (Mohamed, Basant, y Larion, 2017).

9. Efecto del entrenamiento en intervalos de alta intensidad con restricción sanguínea en anaeróbicos. (Behi, Amani, Fahey y Afsharnezhad, 2017).

\section{Hipertrofia}

1. ¿La restricción del flujo sanguíneo mejora la señalización hipertrófica en el músculo esquelético? (Meyer, 2006).

2. Revisión de los procesos de hipertrofia muscular inducida por el entrenamiento de fuerza oclusiva. (Slysz, Stultz, y Burr, 2016).

3. La hipertrofia con ejercicio de resistencia unilateral ocurre sin aumentos en la concentración de la hormona anabólica endógena. (Wilkinson, y Cols., 2006).

4. Ejercicio y flujo sanguíneo de restricción. (Pope, Willardson, y Schoenfeld, 2013).

5. ¿El daño muscular inducido por el ejercicio juega un papel en la hipertrofia del músculo esquelético? (Schoenfeld, 2012).

6. El uso del entrenamiento de oclusión para producir hipertrofia muscular. (Loenneke, Pujol, y Joseoh, 2009).

\section{Seguridad u daños}

1. ¿La restricción del flujo sanguíneo resulta en daño del músculo esquelético? Una revisión crítica de la evidencia disponible. (Loenneke, Thiebaud, \& Abe, 2014)

2. Posibles problemas de seguridad con el entrenamiento de restricción de flujo sanguíneo. Revista escandinava de medicina y ciencia en el deporte. (Loenneke, Wilson, Wilson, Pujol, y Bemben., 2011).

3. Entrenamiento de restricción del flujo sanguíneo y el reflejo presor del ejercicio: una llamada a la preocupación. (Spranger, Krishnan, Levy, O'Leary, y Smith, 2015).

\section{Salud}

1. La restricción del flujo sanguíneo previene el daño muscular, pero no la señalización de la síntesis de proteínas después de las contracciones excéntricas. (Sudo, Ando, Poole, \& Kano, 2015).

2. Rehabilitación del paciente con lesión del ligamento

cruzado anterior de la rodilla (LCA). (Álvarez, López Segovia, Martínez, Legido, 2008).

3. Aplicaciones de la oclusión vascular para disminuir la atrofia por desuso de los músculos extensores de la rodilla. (Takarada, Takazawa, y Ishii, 2000).

4. Ejercicio restringido de flujo sanguíneo y músculo esquelético. (Manini, y Clark, 2009).

5. Eficacia de la isquemia del torniquete para el entrenamiento de la fuerza con baja resistencia. (Shinohara, Kouzaki, Yoshihisa, Fukunaga, 1998).

6. Modelos de progresión en el entrenamiento de resistencia para adultos sanos. (Ratamess, Albar, Evetoch, Housh, Kibler, Kraemer \& Triplett, 2009). 


\section{Hormonal}

1. Comparación de las respuestas hormonales después de la luz ejercicio de resistencia con oclusión vascular parcial y resistencia moderadamente difícil ejercicio sin oclusión. (Reeves, Kraemer, Hollander, Clavier, Thomas, Francois \& Castracane, 2006).

2. Aumento rápido de la hormona de crecimiento plasmática después del ejercicio de resistencia de baja intensidad con oclusión vascular. (Takarada, Nakamura,Aruga, Onda, Miyazaki, y Ishii, 2000).

\section{Deportes}

1. El entrenamiento de oclusión aumenta la fuerza muscular en la división IA jugadores de fútbol. (Yamanaka, Farley, \& Caputo, 2013).

2. Efectos del entrenamiento de resistencia con poca carga combinados con la restricción del flujo sanguíneo o hipoxia en la función muscular y el rendimiento en atletas de baloncesto. (Manimmanakorn, Manimmanakorn, Taylor, Draper, Billaut, Shearman, \& Hamlin, 2013).

3. El uso del entrenamiento de restricción del flujo sanguíneo en un nadador de élite lesionado en el rendimiento de la natación y la hipertrofia. (Morris, 2018).

\section{DISCUSIÓN}

El entrenamiento con restricción de flujo sanguíneo, representa un método eficaz a la hora de generar hipertrofia con cargas bajas $y$ en un menor tiempo comparado con el entrenamiento de fuerza tradicional (Manimmanakorn y col., 2013).

Se han realizado investigaciones sobre los efectos que se obtienen luego de realizar el entrenamiento de restricción de flujo sanguíneo en personas jóvenes entrenados y no entrenados, con distintos parámetros de entrenamiento, es decir, cada investigación utiliza diferentes porcentajes de carga, las cuales varían entre el 20\% del 1 RM (Yasuda, Loenneke, Thiebaud y Abe, 2012), hasta un máximo del 50\% del 1 RM. También difieren en el volumen de series y repeticiones, ya que Yamanaka, Farley y Caputo, (2013) utilizan 4 series de 30 repeticiones, mientras Manimmanakorn y col. (2013) utilizan 3 series al fallo volitivo. Esto supone una problemática ya que, a pesar de reportar beneficios en el aumento de la hipertrofia muscular y de la fuerza, como en este artículo donde demostraron aumentos del $1 \mathrm{RM}$ en press de banca $y$ en sentadillas $(7,0$ y $8,0 \%$, respectivamente), y aumentos en las circunferencias del tórax superior e inferior (3 y 3\%, respectivamente). (Yamanaka, Farley y Caputo, 2013), diversos autores aún no se han puesto de acuerdo en determinar la manera más idónea de realizar el entrenamiento de restricción de flujo sanguíneo, estableciendo parámetros en concretos de entrenamiento.

También se hace mención a cerca de las posibles respuestas, del porque hay aumento prominente de la masa muscular, en la parte endocrina, morfológica, fisiológica, contrastando con los métodos tradicionales, sin embargo, solo son posibles explicaciones, ya que tampoco se ha llegado a determinar cómo actúa el entrenamiento oclusivo para generar hipertrofia y fuerza.

Este último, también representa un problema, porque, al no tener información suficiente, no se puede determinar con certeza y exactitud los mecanismos de 
hipertrofia y aumento de fuerza con el entrenamiento con restricción de flujo sanguíneo, abriendo así, una brecha o un camino bastante amplio para poder seguir haciendo investigaciones en esta área.

Además, muy pocos estudios han tenido un período de entrenamiento que superen el año, para poder establecer los beneficios o prejuicios en un futuro más lejano, orientado a mejorar la hipertrofia muscular, y por supuesto, porque no, la salud misma de la persona. Las variables para la hipertrofia muscular con este método se desconocen aún, es decir, no se ha esclarecido con exactitud los factores que influyen en el aumento de la masa muscular con el entrenamiento de restricción de flujo sanguíneo (Schoenfeld, 2010).

Actualmente, no se ha llegado a proponer un diseño de entrenamiento estándar para el entrenamiento con restricción de flujo sanguíneo, porque en los estudios que se han realizado, los diseños de volumen y cargas varían dependiendo del autor y del caso.

Para poder determinar el nivel de presión al que se debe someter la articulación, Sumide et al. (2009), realizaron un estudio buscaron investigar la presión óptima para ser usada en el entrenamiento de restricción del flujo sanguíneo vascular. El estudio se realizó con veintiún sujetos divididos aleatoriamente en cuatro grupos según la presión aplicada a través de la restricción del flujo sanguíneo vascular. Hubo un grupo sin presión $(0 \mathrm{mmHg})$, un grupo con 50 de presión (50 $\mathrm{mmHg}$ ), un grupo con 150 de presión (150 $\mathrm{mmHg}$ ) y un grupo con $250 \mathrm{de}$ presión (250 $\mathrm{mmHg}$ ).

Cada grupo entrenó 3 veces a la semana durante 8 semanas al $20 \%$ de 1 -RM que realiza levantamiento de pierna recta, aducción de articulación de cadera y entrenamiento de abducción de fuerza máxima. El estudio utilizó la contracción isocinética a 180 grados por segundo para determinar el trabajo muscular (Nm). Los resultados mostraron un aumento significativo en el trabajo muscular en el grupo de presión de $50 \mathrm{mmHg}$ y presión de $150 \mathrm{mmHg}$, lo que sugiere que la presión óptima para el entrenamiento de restricción del flujo sanguíneo vascular está entre 50 y $150 \mathrm{mmHg}$.

De igual manera, la presión usada en el torniquete, es muy variable a lo largo de los estudios. Sin embargo, el análisis del estudio realizado por Slysz, Stultz, y Burr, (2016), indica un rango de presiones entre $50 \mathrm{mmHg}$ hasta $240 \mathrm{mmHg}$ para que se den beneficios.

\section{CONCLUSIÓN}

Como se ha mencionado anteriormente, se han realizado estudios sobre los efectos del entrenamiento de restricción de flujo sanguíneo, pero en varios de estos estudios, utilizan cargas de entrenamiento que varían ampliamente, permitiendo una gran flexibilidad para poder programar diferentes cargas de entrenamiento, sin embargo, esto no permite establecer la manera más idónea de entrenar para obtener el mayor desarrollo de la masa muscular y de la fuerza. (Laurentino et al. 2008). Por lo que los elementos para el entrenamiento quedan establecidos en este rango: Duración: 3 a 16 semanas; frecuencia de 2 a 4 veces por semana; intensidad: 20 - 50\% del 1 RM; descanso: 30 segundos - 60 segundos; presión del torniquete: $50 \mathrm{mmHg}$ - 240 mmHg; series: 3 - 5 por ejercicio; repeticiones: altas, entre 15 a 30, incluso más (al fallo).

Entre los principales mecanismos de adaptación que se han planteado para 
explicar los efectos del entrenamiento con restricción de flujo sanguíneo, se propone: Reclutamiento preferido o adicional de fibras musculares de intercambio rápido, el cual explica el cambio de fibras tipo I a tipo II durante el entrenamiento. Mayor duración de la acidosis metabólica derivada de la retención y acumulación de protones $\mathrm{H}+$, trayendo consigo estrés metabólico. Aumento de la hormona de crecimiento tras el entrenamiento, junto con el aumento de la síntesis de proteínas musculares y de la señalización de mTOR (una enzima responsable de la síntesis de proteínas musculares), y con una mayor activación de las células satélite. Disminución los niveles de miostatina, de la hormona cortisol y de la creatina quinasa.

Es evidente que no hay consenso sobre cuál de estos mecanismos es responsable de las principales adaptaciones del entrenamiento de restricción de flujo sanguíneo, aunque lo más probable es que todos los mecanismos anteriores contribuyan, generando un ambiente anti catabólico, y propicio para el anabolismo.

El entrenamiento con restricción de flujo sanguíneo, o Kaatsu en su país natal en Japón, queda en claro que es un método muy eficaz a la hora de mejorar la hipertrofia muscular, saliéndose de lo tradicional y dejando a un lado los métodos clásicos que, si bien han funcionado y siguen funcionando, deja una interrogante ¿habrá otras formas de entrenar que puedan mejorar el rendimiento deportivo de manera natural y en un periodo de tiempo menor?

Aún falta por descubrir los efectos de este entrenamiento a largo plazo, es decir, después de realizarlo durante por lo menos un año ¿estos beneficios alcanzados cuánto tardarán en mantenerse? O ¿se podrá mantener las ganancias volviendo a utilizar métodos clásicos? O ¿será necesario seguir entrenando con este método para mantener las ganancias obtenidas? También hay que aclarar que a pesar de que los estudios se han realizado en pacientes aparentemente sanos, la recomendación de uso está dirigido precisamente a este tipo de población.

La investigación futura sobre el entrenamiento de oclusión debe enfocarse en estudiar los riesgos para la salud asociados con el uso a largo plazo y determinar las poblaciones en las que este tipo de entrenamiento puede estar contraindicado (Cook, Clark, and PloutzSnyder, 2007). Aunque la investigación aún tiene que definir las poblaciones en las que el entrenamiento de la oclusión es peligroso, se postula que las personas con disfunción endotelial no deben utilizar el entrenamiento de oclusión debido a la reducción en el flujo sanguíneo. La investigación también debería estudiar más a fondo el micro daño a los vasos sanguíneos y los cambios sutiles en el flujo sanguíneo, los cuales pueden estimular la trombosis. Finalmente, los estudios deberían comenzar a enfocarse en los reguladores locales del crecimiento muscular, como los factores de crecimiento.

\section{PROPUESTA Y RECOMENDACIONES PRÁCTICAS}

Como se ha determinado, los autores aún no han concretado una manera idónea de entrenar con el método de restricción de flujo sanguíneo, y a pesar de haber establecido varios parámetros de uso, estos son muy amplios. Por lo tanto, teniendo en cuenta el respaldo de las investigaciones que indican parámetros de seguridad y de efectividad, se propone un modelo de entrenamiento, en donde va dirigido para aquellas personas que 
buscan la hipertrofia muscular y aumento de la fuerza, recalcando las recomendaciones a tener en cuenta para estar dentro de los parámetros de seguridad.

- Como los torniquetes solo pueden ser usados en extremidades, este entrenamiento solo puede ser influido directamente en músculos de tales extremidades, (bíceps, tríceps, flexores y extensores de la mano, el cuádriceps, el femoral y la pantorrilla).

- Aunque el ejercicio en primera instancia se realiza con ejercicios de asilamiento (curl de bíceps, press francés), perfectamente se puede combinar con ejercicios compuestos (press de banca, sentadilla, dominadas).

- Pautas generales de entrenamiento

\begin{tabular}{|l|l|}
\hline Intensidad de la carga: & Entre 20\% y 30\% 1 RM. \\
\hline $\begin{array}{l}\text { Series por grupo } \\
\text { muscular: }\end{array}$ & De 3 a 5. \\
\hline Repeticiones por serie : & $\begin{array}{l}\text { De } 30 \text { a } 40 \text { la primera, 15 } \\
\text { el resto. }\end{array}$ \\
\hline Descanso entre series : & Entre 30 y 60 segundos. \\
\hline Cadencia : & $\begin{array}{l}\text { 2 segundos de concéntrica } \\
\text { y de excéntrica. }\end{array}$ \\
\hline $\begin{array}{l}\text { Tiempo máximo con el } \\
\text { torniquete: }\end{array}$ & De 8 a 10 minutos. \\
\hline
\end{tabular}

Tabla 5 Recomendaciones prácticas

Fuente: Elaboración propia

- El torniquete debe ser retirado cada vez que se confluya un ejercicio, procurando no superar los 10 minutos en uso.

- El torniquete permanecerá en uso entre el descanso de serie y serie del mismo ejercicio.

- El entrenamiento se puede realizar hasta el fallo volitivo.

- Suspender la ejecución del ejercicio si se experimenta falta de aire, vértigo $u$ otros malestares.
- La presión que deben ejercer los torniquetes es desde un rango de $50 / \mathrm{mmHg}$ hasta $160 / \mathrm{mmHg}$, teniendo en cuenta esto, se puede optar por una presión intermedia de 100 a $110 \mathrm{~mm} / \mathrm{Hg}$.

- Realizarlo siempre cuando la persona está en un buen estado de salud.

- Se recomienda la siguiente progresión, considerando dos sesiones kaatsu por semana con pesos entre el 20 y el $30 \%$ de $1 \mathrm{RM}$.

\begin{tabular}{|l|l|}
\hline Semana 1: & $\begin{array}{l}\text { Pruebas de presión, después 1 serie } \\
\text { de } 30 \text { reps. }\end{array}$ \\
\hline $\begin{array}{l}\text { Semana 2-3 } \\
:\end{array}$ & $\begin{array}{l}3 \text { series de } 30 / 15 / 15 / 15 \text { reps, } \\
\text { descansando un minuto entre series. }\end{array}$ \\
\hline Semana 4: & $\begin{array}{l}4 \text { series de } 30 / 15 / 15 / 15 r e p s, \\
\text { descansando 45 segundos entre } \\
\text { series. }\end{array}$ \\
\hline
\end{tabular}

Tabla 6 Recomendaciones prácticas Fuente: Elaboración propia.

Después de la adaptación, se puede optar por protocolos más rigurosos.

\begin{tabular}{|l|l|}
\hline Serie 1: & $\begin{array}{l}3 \text { series de 30/15/15/15 reps con el } \\
20 \% \text { de 1 RM. Descansando 40 } \\
\text { segundos entre series. }\end{array}$ \\
\hline Series 2 a 4: & $\begin{array}{l}4 \text { series de 30/15/15/15reps con el } \\
30 \% \text { de 1 RM, 30 segundos entre } \\
\text { serie y serie. }\end{array}$ \\
\hline Series 5 a 8: & $\begin{array}{l}4 \text { series al fallo, con el 40\% 1 RM, 30 } \\
\text { segundos entre serie y serie. }\end{array}$ \\
\hline Series 9 a 12: & $\begin{array}{l}\text { 5 series al fallo, con el 50\% 1 RM, 45 } \\
\text { segundos entre serie y serie. }\end{array}$ \\
\hline
\end{tabular}

Tabla 7 Recomendaciones prácticas Fuente: Elaboración propia

Para apreciar los resultados luego del programa de entrenamiento, puede optar por realizar una medición de circunferencia de la extremidad y el test de la repetición máxima, con lo que puede verse reflejado el aumento de la masa muscular y de la fuerza. 


\section{REFERENCIAS BIBLIOGRÁFICAS}

Abe, T. \& Beekley et al. (2005a). "Day-to-day change in muscle strength and MRI measure skeletal muscle size during 7 days KAATSU resistance training: A case study." Int. J. KAATSU Training Res. $1,71-76$.

Abe, T. \& M.D. Beekley et al. (2005a). "Day-to-day change in muscle strength and MRImeasure skeletal muscle size during 7 days KAATSU resistance training: A case study." Int. J. KAATSU Training Res. 1, 71-76

Abe, T., Kearns, C., Sato, Y. (2006). Muscle size and strength are increased following walk training with restricted venous blood flow from the leg muscle, Kaatsu-walk training. J Appl Physiol.;100(5): P.P. 1460-6pp.

Abe, T., Sakamaki, M., Fujita, S., Ozaki, H., Sugaya, M., Sato, Yoshiaki, M., \& Nakajima, Toshiaki, M. (2010). Effects of low-intensity walk training with restricted leg blood flow on muscle strength and aerobic capacity in older adults. Journal of Geriatric Physical Therapy, 33(1), 34-40.

Abe, T.; Charles, F.; Kearns, \& Sato, Y. (2005). Muscle size and strength are increased following walk training with restricted venous blood flow from the leg muscle, Kaatsu-walk training. Journal of Applied Physiology, 100(5), 1460-1466.

ACSM. (2009). American College of Sports Medicine position stand. Progression models in resistance training for healthy adults. Med Sci Sports Exerc.; 41(3), P. 687-708.

Alfonso, A. G. (2017). La escritura científica: Una revisión temática. Signo y Pensamiento, 36(71), 52

Álvarez, R., López-Silvarrey, J., Segovia, C., Martínez, H., y

Legido-Arce, J. (2008). Rehabilitación del paciente con lesión del ligamento

cruzado anterior de la rodilla (LCA). Revisión. Revista Internacional de Medicina y

Ciencias de la Actividad Física y el Deporte, 8(29), 62-92.

Baechele, Earle. (2007). Principios del entrenamiento de la fuerza y del acondicionamiento físico. National Strength and Conditioning Association

Barnett, E., Dankel, J., Counts, R., Nooe, A., Abe, T., Loenneke, J. (2016). Blood flow occlusion pressure at rest and immediately after a bout of low load exercise. Clinical Physiology and Functional Imaging, Pages 436-440.

Behi, A., Amani, A., Fahey, T., \& Afsharnezhad, T. (2017). Effect of high intensity interval training with blood restriction on anaerobic performance.International Journal of Applied Exercise Physiology, 6(2), 45-52.

Brandner, C., Warmington, S., \& Kidgell, D. (2015). Corticomotor Excitability is Increased Following an Acute Bout of Blood Flow Restriction Resistance Exercise. Frontiers in human neuroscience, 9.

Campo, C., Granados, F., Muñoz, L., Sergio, G., \& Rodríguez S. (2012). Caracterización del avance teórico, investigativo y/o de intervención en resiliencia desde el ámbito de las universidades en Colombia. Universitas Psychologica, 11(2), 545-557.

Cook, S., Clark, B., and Ploutz-Snyder, L. (2007). Effects of exercise load and blood-flow restriction on skeletal muscle function. Med Sci Sports Exerc 39: 1708-1713

De Castro, P., Aquino, R., Berti, A., Gonçalves, C., \& Puggina, F. (2017). Strength Training with Vascular Occlusion: A Review of Possible Adaptive Mechanisms. Human Movement, 18(2), 3-14. 
Guajardo, E., \& Castro, A. (2007). la investigación cualitativa, una discusión presente. Liberabit, 13, 63-69.

Gürtler, L., \& Huber, G. (2007). modos de pensar y estrategias de la investigacion cualitativa. Liberabit, 13, 37-52.

Hernández, M., Azael, J. (2012) Respuestas y adaptaciones al entrenamiento de fuerza oclusivo de baja intensidad. Revista Digital. Buenos Aires, № 164.

Hernández, R., Fernández, C., \& Baptista, P. (2010). Metodología de la investigación. México, D.F., México.: Mc. Graw Hill.

Ingram, W., Dankel, J., Buckner, L., Counts, R., Mouser, G., Abe, T., y Loenneke, P. (2017). The influence of time on determining blood flow restriction pressure. Journal of Science \& Medicine in Sport, 20(8), 777-780.

Karabulut, M., Leal, A., Garcia, D., Cavazos, C., \& Bemben, M. (2014). Tissue oxygenation, strength and lactate response to different blood flow restrictive pressures. Clinical Physiology \& Functional Imaging, 34(4), 263-269.

Karabulut, M., Mccarron, J., Abe, T., Sato, Y., y Bemben, M. (2011). The effects of different initial restrictive pressures used to reduce blood flow and thigh composition on tissue oxygenation of the quadriceps. Journal of Sports Sciences, 29(9), 951-958.

Kubo, K., Komuro, T., Ishiguro, N., Tsunoda, N., Sato. Y., Ishii, N., et al. (2006). Effects of low-load resistance training with vascular occlusion on the mechanical properties of muscle and tendon. J Appl Biomech.;22; pp. 112-9.

Laurentino, G., Ugrinowitsch, C, Aihara, Y., Fernandes, R., Parcell, C., Ricard, M., et al (2008) Effects of strength training and vascular occlusion. Int J Sports Med.29; pp. 664-7.

Loenneke, J., \& Pujol, T., Joseoh, E. (2009). The use of occlusion training to produce muscle hypertrophy. Strength and Conditioning Journal, 31(3), 77-84.

Loenneke, P., Thiebaud, S., \& Abe, T. (2014). Does blood flow restriction result in skeletal muscle damage? A critical review of available evidence. Scandinavian journal of medicine \& science in sports,24(6), e415-422.

Loenneke, P., Wilson, J., y Wilson, M. (2010). A Mechanistic Approach to Blood Flow Occlusion. International Journal of Sports Medicine, 31(1), 1-4.

Loenneke, P., Wilson, M., Wilson, J., Pujol, J., \& Bemben., G. (2011). Potential safety issues with blood flow restriction training. Scandinavian journal of medicine \& science in sports, 21(4), P.P.510-518.

Madarame, H., Neya, M., Ochi, E., Nakazato K, Sato, Y., and Ishii, N. (2008). Crosstransfer effects of resistance training with blood flow restriction. MedSciSportsExerc 40; pp. 258263.

Manimmanakorn, A., Hamlin, M., Ross, J., Taylor, R., \& Manimmanakorn, N. (2013). Effects of lowload resistance training combined with blood flow restriction or hypoxia on muscle function and performance in netball athletes.Journal of Science and Medicine in Sport, 16(4), 337-42.

Manimmanakorn, A., Manimmanakorn, N., Taylor, R., Draper, N., Billaut, F., Shearman, J., \& Hamlin, M. (2013). Effects of resistance training combined with vascular occlusion or hypoxia on neuromuscular function in athletes. European Journal of Applied Physiology, 113(7), 1767-1774. 
Manini, M., \& Clark, C. (2009). Blood Flow Restricted Exercise and Skeletal Muscle Health. Sport Sciences Reviews, 37(2), 78-85.

Martín J., Marín, J., Menéndez, P., Ferrero, C., Loenneke, P., \& Herrero, A. (2013a). Muscular adaptations after two different volumes of blood flowrestricted training. Scandinavian Journal of Medicine and Science in Sports, 23(2), 114-120.

Martín, J., Marín, J., y Herrero, J. (2011) Revisión de los procesos de hipertrofia muscular inducida por el entrenamiento de fuerza oclusivo. Rev Andal Med Deporte.;4(4); pp. 152-157.

Meyer R (2006). Does blood flow restriction enhance hypertrophic signaling in skeletalmuscle?. J pplPhysiol 100: 1443-1444.

Mohamed, S., Basant, S., \& Larion, A. (2017). Effect of Kaatsu Training on Serum Protein Electrophoresis and Performance Level of 100M Hurdles for Female College Students. Ovidius University Annals, Series Physical Education \& Sport/Science, Movement \& Health, 17(2), 122 128

Moore, D., Burgomaster, K., Schofield, L., Gibala, M., Saie, D., and Phillips, S. (2004). Neuromuscular adaptations in human muscle following low intensity resistance training with vascular occlusion. Eur J Appl Physiol 92: 399-406

Moritani, T., Sherman, W., Shibata, M., Matsumoto, T., Shinohara M. (1992). Oxygen availability and motor unit activity in humans. Eur J Appl Physiol Occup Physiol, 64:552-6.

Morris, N. (2018). the use of blood flow restriction training in an injured elite swimmer on swimming performance and hypertrophy. Journal of Australian Strength \& Conditioning, 26(1), 31-36.

Nakajima, T., lida, H., Kurano, M., Takano, H., Morita, T., Meguro, K., Abe, T. (2008). Hemodynamic responses to simulated weightlessness of 24-h head-down bed rest and KAATSU blood flow restriction. European Journal of Applied Physiology, 104(4), 727-37

O'Halloran, J. (2014). The hypertrophic effects of practical vascular blood flow restriction training (Order No. 1555232).

Patterson, S. D., \& Ferguson, R. A. (2010). Increase in calf post-occlusive blood flow and strength following short-term resistance exercise training with blood flow restriction in young women. European Journal of Applied Physiology, 108(5), 1025-33.

Patterson, S., Ferguson, A. (2010) Increase in calf post-occlusive blood flow and strength following short-term resistance exercise training with blood flow restriction in young women. Eur J Appl Physiol.;108; pp. 1025-33.

Pope, K., Willardson, M., \& Schoenfeld, J. (2013). Exercise and blood flow restriction. Journal of Strength and Conditionating Research, 27(10), 2914-2926.

Ratamess, A., Albar, A., Evetoch, K., Housh, J., Kibler, W., Kraemer, J., \& Triplett, T. (2009). Special Communication. American College of Sports Medicine Position Stand: Progression models in resistance training for healthy adults. Medicine and Science in Sports and Excercise, 41(3).

Reeves, V., Kraemer, R., Hollander, D., Clavier, J., Thomas, C., Francois, M., \& Castracane, D. (2006). Comparison of hormone responses following light resistance exercise with partial vascular occlusion and moderately difficult resistance exercise without occlusion. Journal of Applied Physiology, 101(6), 1616-1622. 
Reyes, J. (2013). una experiencia en la enseñanza de la investigación educativa en el marco de "proyecto aula" de la universidad veracruzana. Revista Mexicana De Investigación Educativa, 18(58), 735-768.

Schoenfeld, B. (2010). the mechanisms of muscle hypertrophy and their application to resistance training. Journal Of Strength \& Conditioning Research (Lippincott Williams \& Wilkins), 24(10), 2857-2872.

Schoenfeld, J. (2012). Does exercise-induced muscle damage play a role in skeletal muscle hypertrophy? J. Strength Cond. Res. 26; pp.1441-1453.

Shinohara, M., Kouzaki. M., Yoshihisa, T., Fukunaga, T. (1998). Efficacy of tourniquet ischemia for strength training with low resistance. European J Appl Physiol Occup Physiol.; 77; pp. 189-91.

Silva, G., Aniceto, R., Oliota-Ribeiro, S., Neto, R., Leandro, S., \& Cirilo-Sousa, S. (2018). Mood Effects of Blood Flow Restriction Resistance Exercise Among Basketball Players. Perceptual \& Motor Skills, 125(4), 788-801.

Slysz, J., Stultz, J., \& Burr, J. (2016). The efficacy of blood flow restricted exercise: A systematic review \& meta-analysis. Journal Of Science \& Medicine In Sport, 19(8), 669-675

Spranger, D., Krishnan, C., Levy, D., O'Leary, S., \& Smith, A. (2015). Blood flow restriction training and the exercise pressor reflex: a call for concern. American Journal of Physiology-Heart and Circulatory Physiology, 309(9), H1440-H1452.

Subiela, J. V., \& Torres, S. H. (2010). Aportaciones de la biopsia muscular al entrenamiento deportivo. / Contributions of muscle biopsy to sport training. Revista Kronos, 9(18), 21-30.

Sudo, M., Ando, S., Poole, C., \& Kano, Y. (2015). Blood flow restriction prevents muscle damage but not protein synthesis signaling following eccentric contractions. Physiological reports, 3(7), e12449.

Sumide, T, Sakuraba, K, Sawaki, K, Ohmura, H, and Tamura, Y. (2009). Effect of resistance exercise training combined with relatively low vascular occlusion. J Sci Med Sport 12: 107-112.

Takarada, Y., Nakamura, Y., Aruga, S., Onda, T., Miyazaki, S., Ishii, N. (2000). Rapid increase in plasma growth hormone after low intensity resistance exercise with vascular occlusion. J Appl Physiol 88: 61:65

Takarada, Y., Takazawa, H., \& Ishii, N. (2000a). Applications of vascular occlusion diminish disuse atrophy of knee extensor muscles. Medicine and Science in Sports and Excercise, 32, 2035-2039

Triviño, A.,Isabel Bernal. (2008). Preferencias de la información en internet. una nueva propuesta metodológica, ensayada con un grupo de universitarios/Preferences design information on the internet. A new methodological proposal, carried out with the university students. Revista Latina De Comunicación Social, (63), 189-216.

Warnock, E., \& Bachman, R. (2015). Health \& wellness: A kaatsu promise: A workout with bigger results. Wall Street Journal.

Warnock, E., \& Bachman, R. (2015). In japan, can you work out less, get more results? --- A training technique called kaatsu mixes light exercise with pressurized cuffs. Wall Street Journal

Weatherholt, A., Beekley, M., Greer, S., Urtel, M., \& Mikesky, A. (2013). Modified Kaatsu Training: Adaptations and Subject Perceptions. Medicine and Science in Sports and Exercise, 45(5). 
Wilkinson, S., y Cols. (2006). Hypertrophy with unilateral resistance exercise occurs without increases in endogenous anabolic hormone concentration. Eur J Appl Physiol. 2006; 98 (6); pp. 546-55pp.

Wolinski, P., Neves, E., \& Pietrovski, E. (2013). Analysis of hemodynamic and vascular repercussions of kaatsu training. ConScientiae Saúde, 12(2), 305-312.

Yamanaka, T., Farley, R., \& Caputo, L. (2013). Occlusion training increases muscular strength in división IA football players. Journal of Strength and Conditioning Research, 26(9), 2523-2529.

Yasuda, T., Loenneke, P., Thiebaud, S., \& Abe, T. (2012). Effects of blood flow restricted low-intensity concentric or eccentric training on muscle size and strength. Plos one, 7(12), e52843.

Yasuda, T., Loenneke, P., Thiebaud, S., \& Abe, T. (2015). Effects of detraining after blood flowrestricted low-intensity concentric or eccentric training on muscle size and strength. The Journal of Physiological Sciences, 65(1), 139-144. 